\title{
De la autonomía del mandato a la rendición de cuentas. Un alcance conceptual a los mecanismos de representación democrática
}

\author{
Octavio Avendaño ${ }^{1}$
}

\section{Resumen}

Este artículo tiene como objetivo efectuar una revisión de la noción de representación democrática, a la luz de interpretaciones que en las últimas décadas surgen desde la sociología y la ciencia política. Para ello, se consideran los aspectos teóricoconceptuales y las experiencias históricas más relevantes que se suceden tras la emergencia de la concepción moderna de representación, hasta los debates y situaciones que afectan a las actuales democracias. Para el despliegue y la exposición de los argumentos se presentan dos grandes apartados. El primero, subdividido en cuatro secciones, muestra el paso de las formas y nociones de representación premodernas a las modernas que aparecen hacia fines del siglo XVIII, y que alcanzan su arraigo definitivo con el establecimiento de la democracia liberal. El segundo, también dividido en cuatro secciones, aborda el fenómeno de la representación a partir de las nociones e instituciones que sustentan a las democracias contemporáneas. Se consideran acá los debates más recientes acerca de la calidad del funcionamiento de las instituciones democráticas, el fortalecimiento de la intermediación y los mecanismos de rendición de cuentas.

\section{From the autonomy of mandate towards accountability. A conceptual approach to the mechanisms of democratic representation}

Abstract

This paper aims to review the notion of democratic representation under the light of the latest interpretations emerged from the sociology and political science fields. For doing so, I consider theoretical and conceptual aspects plus the most relevant historical experiences that have happened after the emergence of the modern conception of democratic representation. I equally consider the current debates on the issue affecting democracies. The arguments are presented in two parts. The first one, divided in four sub-sections, shows the transition of the pre-modern notions and forms of representation to the modern ones by the end of the eighteen century. The second part, divided in four sections as well, analyzes the representation phenomenon from the notions and institutions that sustain modern democracies. In this second part, are also considered the latest debates on the quality of the functioning of the democratic institutions, the strengthening of the intermediation and the mechanisms of accountability.

${ }^{1}$ Académico del Departamento de Sociología de la Universidad de Chile (oavendan@uchile.cl).Doctorando en Ciencias Políticas, Università degli studi di Firenze (Italia). El autor agradece los comentarios y sugerencias de los editores, Raimundo Frei y Cristóbal Rovira, además de quienes leyeron las sucesivas versiones del artículo: Emmanuelle Barozet, Mauro Basaure, Daniel Chernilo, Rodrigo Figueroa y Jorge Vergara. 


\section{Presentación}

Son diversos los diagnósticos que en las últimas décadas han caracterizado los niveles de calidad de las democracias y han expuesto las tensiones que ellas presentan. Gran parte de estos diagnósticos se sitúan a nivel de los problemas del funcionamiento de las instituciones. De ahí que fenómenos como la desafección política o el debilitamiento del apoyo popular hacia las instituciones democráticas, sean interpretados como síntomas de crisis en las formas tradicionales de representación, más que como una tensión no resuelta que vienen experimentando las democracias desde el momento mismo de su constitución.

La representación es la base y el fundamento de los sistemas democráticos modernos. Su entramado institucional define las formas de inserción y vinculación con la comunidad política, reconociéndose preferentemente a través del vínculo entre representantes y representados, o mediante la función de intermediación que cumplen las organizaciones que emanan desde el sistema político y la sociedad civil.

El componente esencial de la representación radica en la relación e interacción que establecen dos o más actores por medio del cual uno actúa, o decide, en nombre de otros (Bobbio 1988: 7, Accarino 1999: 19, Cedroni 2004: 20). En el marco de esta relación, los representados hacen uso de instrumentos y formas de participación democrática para ratificar, cambiar o simplemente escoger a quienes actúan en su nombre a nivel del gobierno o en otras esferas deliberativas. A medida que la sociedad se va haciendo más compleja y diferenciada, y se van ampliando las posibilidades de participación, se acentúa la despersonalización de este vínculo y aumenta la autonomía de parte de los representantes.

Uno de los momentos decisivos, que se ubica hacia fines del ancien régime, corresponde a la adopción del principio de la autonomía del representante, a través del cual se pretende superar aquel mandato imperativo prevaleciente en los sistemas representativos de la fase premoderna. El principio de autonomía entra en concordancia con una serie de otros postulados puestos en boga en este mismo período, que apuntan a garantizar una adecuada separación de los poderes y mecanismos de control por parte de los ciudadanos. Sin embargo, la promoción y la defensa que se hace de dicho principio, conlleva desde muy temprano el riesgo de producir la independencia absoluta de los representantes, junto a la ausencia de control por parte de los representados. El riesgo de esta separación, o de disolución del vínculo que fundamenta el mandato de los representantes, constituye uno de los principales focos de preocupación para el devenir de las actuales democracias.

Es por eso que este artículo se concentra en la tensión que genera el vínculo de la representación democrática, poniendo un especial énfasis en las formas de represen- 
De La AUTONOMÍA DEL MANDATO A LA RENDICIÓN DE CUENTAS. UN ALCANCE CONCEPTUAL A LOS MECANISMOS DE REPRESENTACIÓN DEMOCRÁTICA

tación electivas que se dan al interior de los sistemas democráticos. Se parte del supuesto que los sistemas democráticos deben lograr la articulación entre un mandato despersonalizado y autónomo, con el desarrollo de formas de control ciudadano que garanticen la responsabilidad por parte de los representantes. La desarticulación, en cambio, podrá derivar en una excesiva concentración del poder en la figura de los representantes y en la pérdida absoluta de la soberanía por parte de los ciudadanos - como se ha venido constatando en varias de las "nuevas democracias" (O'Donnell 1994). En consecuencia, la autonomización del mandato asegura consistencia y viabilidad a un sistema democrático, en la medida que ésta sea regulada y controlada desde los propios ciudadanos.

Para los efectos de este artículo, la lectura, y al mismo tiempo la escritura de estos fenómenos, ha sido hecha en clave institucional, sin desconocer la importancia que puedan tener aspectos de carácter simbólico a nivel de las diferentes formas de representación. La presentación y el orden de los argumentos se realizan en función de dos grandes apartados. El primero de ellos, subdividido en cuatro secciones, se concentra en la defensa y la adopción de la idea moderna de representación, a partir de la disolución de las formas premodernas, asociadas a la representación de intereses y al mandato imperativo. Junto a la descripción de los aspectos institucionales que sustentan los sistemas representativos que se van configurando, en esta primera parte se da cuenta de los planteamientos derivados de dos exponentes del liberalismo clásico: Edmund Burke y John Stuart Mill. La segunda parte, en cambio, da cuenta de los principales desafios que asumen las democracias contemporáneas, para asegurar la vigencia de los mecanismos de representación y por ende su propia sostenibilidad en el tiempo. En esta segunda parte se toman en cuenta algunos de los debates más importantes que derivan del fenómeno de la democratización, de los problemas de la participación y de la competencia partidaria.

\section{El sistema representativo en la configuración de las demo- cracias modernas.}

\section{I.1. El significado premoderno de la representación}

La idea de representación no es una derivación directa de la democracia moderna, sino de un conjunto de prácticas e instituciones cuyo origen se remonta a la fase premoderna. Más específicamente, es de origen medieval, aunque desde el punto de vista jurídico existen antecedentes que se encuentran en normativas y disposiciones establecidas ya en el derecho romano, el cual contempla por primera vez que juristas y magistrados puedan interceder o actuar en nombre de otros. Pero es durante el 
medioevo en que se dan las condiciones institucionales para la emergencia de formas de representación, muchas de ellas de carácter local que hacen posible la distribución y la participación en el poder político (Poggi 1978: 71ss, Mann 1991). Surgen ahí una serie de prácticas y agentes - portavoces, abogados y embajadores - destinados a actuar en función de los intereses de determinadas clases, corporaciones y gremios. Se origina, de este modo, una representación de intereses (corporativos) que se mantiene a pesar de la disolución de algunas instituciones feudales durante el resto de la fase premoderna ${ }^{2}$.

Para llevar a cabo esta forma de representación, durante todo este período se ponen en práctica la convocatoria y la participación en asambleas de carácter local, u otras con una cobertura territorial más amplia como ocurre con el parlamento. La disgregada presencia de las asambleas locales y provinciales - y en especial la presencia del parlamento -, contribuyen durante el medioevo a descentralizar el poder y a limitar las capacidades de acción de la propia monarquía, al punto que ésta se ve obligada a consultar a las asambleas sobre una serie de asuntos relacionados con impuestos y el despliegue de las milicias. Mientras el alto clero y la alta nobleza se representan interviniendo personalmente, la baja nobleza, el bajo clero y la burguesía urbana lo hacen enviando representantes que tutelan y defienden los intereses de cada uno de estos sectores. Durante todo este período, la selección y definición de los representantes se realiza por mecanismos no electivos, como la designación, la cooptación o la herencia del cargo (Cotta 1986: 291). El nivel de institucionalización de estos mecanismos tiende a variar de país en país, aunque prevalece en todos ellos una expresión de pluralidad de intereses y sectores sociales representados, que alcanza un consenso general a través de las decisiones finales del monarca, quien a la vez personifica y representa simbólicamente la nación.

Es sabido que las relaciones entre las asambleas y la monarquía estuvieron en varios momentos marcadas por tensiones, originadas por la pretensión de la corona de tener un control más directo de los sistemas de tributación y de las milicias, o en otros casos, en los cuales existía tradición parlamentaria como en Inglaterra, intentar limitar el poder del parlamento (Mann 1991: 611-613). La disolución del ancien régime y el advenimiento de las formas modernas de representación, no provocan una ruptura total con aquellas instituciones representativas predominantes en la fase premoderna. Como advierte Cotta, la ruptura entre lo premoderno y lo moderno desde el punto de vista de las instituciones representativas se torna más bien ideológica que real (Cotta 1986: 294). De hecho, la tradición parlamentaria y de participación descentralizada se arraiga con más fuerza en aquellos países en los cuales estas formas de representación existen ya en la fase premoderna.Aún así, en el contexto del iluminismo y en especial a partir de las experiencias revolucionarias francesa y americana, se

${ }^{2}$ En este sentido, dice Alessandro Pizzorno: "En cuanto expresión de cuerpos sociales preexistentes a lo político, la representación del ancien régime era esencialmente representación de intereses, que eran defendidos al presentar las demandas hacia el soberano (que eran siempre demandas fiscales)" (Pizzorno 1996: 964). 
De La AUTONOMÍA DEL MANDATO A LA RENDICIÓN DE CUENTAS. UN ALCANCE CONCEPTUAL A LOS MECANISMOS DE REPRESENTACIÓN DEMOCRÁTICA

produce un cambio significativo en la idea de representación sustentada en intereses corporativos y en el mandato imperativo, a partir de la introducción de nuevos principios como la transferencia de la responsabilidad y la deliberación.

\section{I.2. La ruptura con la concepción tradicional: la autonomía del representante}

Parte importante del pensamiento político de la Ilustración, junto a quienes formulan buena parte de las proposiciones que surgen al calor de las dos experiencias revolucionarias aludidas, asumen la idea de representación en contraposición a los postulados, todavía vigentes, favorables a la participación y la democracia directa. Los argumentos esgrimidos a favor de la representación toman en cuenta el carácter heterogéneo y diferenciado que han asumido las sociedades, así como también la presencia de un ordenamiento jurídico y territorial distinto al de la antigüedad clásica y al de las ciudades-Estado del medioevo: el Estado-nación. Con la emergencia del Estado-nación, no resultaba viable un modelo o sistema político que promueva la deliberación directa del conjunto de la población. Este tipo de argumentos aparece con fuerza en los autores de The federalist papers - Madison, Hamilton y Jay -, así como también en Tocqueville y John Stuart Mill, quienes añadían la necesidad de constituir una forma de organización política capaz de representar la diversidad de intereses de la totalidad de la población que forma parte de un determinado territorio. Todos ellos, además, compartían el hecho de concebir la representación a partir de la elección de delegados a los cuales se les transfiere la responsabilidad de la deliberación ${ }^{3}$. Como ya se ha indicado, esto último establece una diferencia sustantiva con la concepción premoderna de representación al hacer del representante un sujeto autónomo respecto a sus propios representados. Se trata de una diferencia que se ve acentuada por el carácter electivo asumido por la representación, especialmente a nivel de los cuerpos legislativos y por la importancia que cobra posteriormente la expansión del sufragio.

En su celebre "Discurso a los electores de Bristol" (1774), Edmund Burke propuso por primera vez un tipo de representación desligada de los intereses de determinados grupos sociales y de las realidades locales. Burke pronunció dicho discurso como reacción a un hecho que se venía dando al interior del parlamento inglés: la permanente presión de los terratenientes por lograr que se legisle a su favor. Por tal

${ }^{3}$ De los autores que inauguran la concepción moderna de la representación, este artículo se concentra únicamente en Edmund Burke y John Stuart Mill. No se consideran acá los autores de The federalist papers, ni tampoco los aportes de Tocqueville, no obstante la reflexión que ellos instalan sobre la descentralización del poder y el peligro que encierra la "tiranía de la mayoría" - cuando no se asegura la representación de las minorías - ya que buena parte de estos argumentos son posteriormente recuperados por el propio Mill. Más bien interesa dar cuenta de cómo Burke define por primera vez el carácter autónomo de la representación y los fundamentos que da Mill para justificar la presencia del gobierno representativo. Además, el tipo de reflexión de Burke y de Mill se sitúa en los momentos de disolución del ancien régime y la configuración de un nuevo orden social y político. 
motivo, consideraba que el parlamento debía ser concebido como una asamblea resolutiva y deliberativa de los asuntos de interés nacional (Burke [1774] 1984:313).Y consecuentemente, correspondía al representante deliberar y decidir teniendo como referencia al conjunto de la nación.

Junto a ello, Burke destacaba el carácter electivo que debe tener la representación, con lo cual establecía una clara separación con aquella forma nominativa y hereditaria prevaleciente en la fase premoderna ${ }^{4}$. Pero además, al representante le correspondía como tarea establecer una comunicación estrecha con sus electores prestando una permanente atención a sus aspiraciones, llegando incluso a sacrificar su reposo y sus placeres si fuese necesario. No obstante esta comunicación, al interior del parlamento cada representante debía actuar y decidir haciendo abstracción de los intereses y las demandas de quienes lo habían elegido como tal. De ahí que la deliberación del representante debía ser ejercida en plena autonomía. Pues si un representante sacrificaba su discernimiento en función de la opinión de los electores, los terminaba a fin de cuentas traicionando más que ayudando o favoreciendo (Accarino 1999: 65).

Esta autonomía planteada por Burke es concebida como el reconocimiento de una nueva clase política, de cuyo desarrollo emerge posteriormente la figura del "político profesional", descrito por Max Weber. En el momento de la disolución del ancien régime, esta nueva clase política ya no goza del "natural control sobre la sociedad local", como antes había ocurrido con los sectores derivados de la nobleza (Pizzorno 1996: 966). Por el contrario, desde sus inicios utilizaba una serie de mecanismos para filtrar las demandas emanadas desde la sociedad. Burke no era explícito al señalar qué ocurría una vez que los electores han transferido la responsabilidad y la capacidad de decisión al representante; ni mucho menos, cómo asegurar que el representante posteriormente decidiera en nombre de ellos. Solamente hacía referencia a la importancia que cobraba la confianza en el marco del vínculo entre representantes y representados. Siguiendo algunas interpretaciones más recientes sobre ese período (Accarino 1999: 67ss), el vínculo de confianza entre ambos debía venir desde arriba hacia abajo y no a la inversa. Después de todo, afirmaba Burke, los electores conocen nada o muy poco de quienes deberán servirles o actuar en nombre de ellos.

Varias de las definiciones entregadas por Burke coinciden con aquellos planteamientos que formularán posteriormente los revolucionarios franceses, especialmente en 1791, que es cuando el proceso se ha encaminado hacia la formación de una monarquía de carácter constitucional. En ese momento, se hacía necesario establecer un mecanismo que permitía representar al conjunto de las facciones y clubes - como el Bretón, el de los Cordeleros y el de los Jacobinos - que participaban activamente del proceso revolucionario. Así como también, se hacía necesario buscar la fórmula que permitía canalizar las distintas posiciones e intereses de los grupos en pugna. La

\footnotetext{
4“El hecho de ser electo - subraya Pizzorno - le da a los representantes aristocráticos una legitimidad a través de la cual se logran contraponer a la legitimidad monárquica” (Pizzorno 1996: 968).
} 
definición propuesta y que se expresaba constitucionalmente, resaltaba también a la nación por sobre cualquier contexto local, como el referente que debía tener todo representante que había sido electo para funciones de orden legislativo ${ }^{5}$. Como se señala en la propia Constitución de 1791, "los representantes nominados en las circunscripciones no representan una particular circunscripción sino a la entera nación" (citado por Sartori 1995: 287).

Los revolucionarios franceses tampoco clarificaban la existencia de garantías ofrecidas por los representantes más allá de la confianza depositada, esta vez, por los representados. En consecuencia:"Se elige a un diputado únicamente porque se tiene confianza en él y porque se le cree capaz, en las cuestiones que se presenten, de no tener ante sí más que el interés del pueblo" (Groethuysen 1993:260). En este contexto, no es posible ver con claridad la presencia de un mecanismo de control. Ni siquiera se reconoce a través del uso del sufragio, por más regularidad que se le intentaba otorgar a las elecciones. En gran medida, eso se debe al carácter dinámico e intenso del propio proceso revolucionario, ya que éste implica resolver en el momento una serie de cuestiones y que obliga a los representados a tener que simplemente confiar en las decisiones adoptadas por sus representantes.

\section{I.3. La representación como distribución y control del poder}

La idea de una representación autónoma es recogida posteriormente por parte importante del liberalismo que emerge, como discurso y como práctica, durante la primera mitad del siglo XIX. Eso sí, no se logra materializar aún en un sentido democrático, ya que los regímenes que la adoptaron - a pesar de la influencia del liberalismo, de la incorporación del parlamentarismo y del constitucionalismo - están lejos de ser considerados democráticos. Sobre todo, si se tiene en cuenta que no se había producido la expansión del sufragio hacia el conjunto de los trabajadores y mujeres. Más aun, la mayoría de las asambleas y parlamentos seguía sin tener atribuciones para derribar o rechazar un mal gobierno (Macpherson 1997). Esta situación comenzó a ser revertida de manera paulatina, hacia mediados del siglo XIX, con la irrupción de las nuevas organizaciones derivadas de la clase trabajadora y el surgimiento de grupos políticos que abogaron por la instauración del sufragio universal. También influyen en esta transformación ciertas reformas políticas que establecían la regularidad de las elecciones para la renovación de las asambleas legislativas y la posibilidad de hacer electiva la conformación del gobierno.

Desde la perspectiva del liberalismo, comenzó a adquirir especial preocupación la necesidad de descentralizar el poder político junto a promover mecanismos que per-

\footnotetext{
${ }^{5} \mathrm{Al}$ igual como había señalado Burke, sostiene Carré de Malberg: "la palabra representación (...) expresa la idea de un poder dado al representante de querer decidir por la nación” (citado por Sartori 1995: 288).
} 
mitían controlar y regular la gestión de las autoridades, con el fin de evitar abusos o la reaparición de situaciones de carácter despóticas. Pero más importante aún será la reflexión que suscitó toda la transformación derivada de la crisis del "orden tradicional" y las revoluciones políticas que se llevaron a cabo en Europa durante la primera mitad del siglo XIX. Precisamente, entre estos acontecimientos encontramos las reflexiones sobre el gobierno y las instituciones representativas que realizó uno de los mayores exponentes del liberalismo decimonónico: John Stuart Mill.

Coherente con la tradición iluminista, Mill veía también al gobierno representativo como una alternativa viable en relación a otras formas más participativas y de democracia directa. A lo cual agregaba, siguiendo esta vez el ejemplo de la Grecia clásica, que la participación y la inserción en la esfera pública - que pueden ser perfectamente inducidas - ayudan a educar a los ciudadanos haciéndolos de ese modo mucho más virtuosos (Mill [1861] 1991: 82-83). Pese a ello, consideraba que en su época existían una serie de limitantes para lograr ese objetivo: la rutina y los problemas cotidianos que enfrentaban las personas tendían a exacerbar el individualismo y la indiferencia frente a lo público; al mismo tiempo, el agobio que provocaba el afán de satisfacer determinadas aspiraciones materiales mermaba todo altruismo, llevándolas a buscar refugio en su propia individualidad o, a lo sumo, en la familia (Ibid: 85). De acuerdo con Mill, la lógica que subyace a la representación permitía, en parte, contrarrestar esta tendencia al entregar a la mayoría de los hombres un nivel mínimo de inserción y preocupación por los asuntos públicos.

Según este autor, existían otros atributos relacionados con las posibilidades que ofrecía la instauración del gobierno representativo para salvaguardar la pluralidad y el ejercicio de la soberanía por parte del pueblo (Ibid: 105). Del lado de la pluralidad, la presencia del gobierno representativo facilitaba la confluencia de aspiraciones e intereses diversos. Del lado de la soberanía, en cambio, la relevancia radicaba en ofrecer que "todo el pueblo, o una gran parte del mismo" expresara su voluntad a través de representantes electos. Para Mill, el representante, además de velar por el interés general de la nación, tenía como función ejercer control sobre todas las operaciones gubernamentales (Ibid). En la medida en que los representantes hacían efectivo el control, las atribuciones y funciones del ejecutivo, éstas se verían limitadas y "fiscalizadas", evitando toda posibilidad de concentración y abuso de poder. Por este motivo, afirmaba que la forma representativa de gobierno garantizaba que la soberanía y las "fuerzas de control", siguieran residiendo en la comunidad o si se prefería, dispersa entre los ciudadanos.

Como se ve, la capacidad de control residía en el representante y no en los ciudadanos o en los electores. Dicho de otro modo, en la óptica de Mill, los ciudadanos no sólo traspasan el ejercicio de la deliberación sino también toda posibilidad de ejercer control directo sobre el gobierno. Si esto es así, ¿quién controlaba entonces 
De La AUTONOMÍA DEL MANDATO A LA RENDICIÓN DE CUENTAS. UN ALCANCE CONCEPTUAL A LOS MECANISMOS DE REPRESENTACIÓN DEMOCRÁTICA

al representante (al controlador)? La respuesta a esta interrogante no se encuentra en Mill, quien tras asignar la función de control al representante se remitía a realizar una suerte de analogía al definir el rol del parlamento. Por un lado, Mill señalaba que la función del parlamento no era gobernar sino "vigilar y controlar" al gobierno o al ejecutivo (Ibid: 123). Junto a ello, debía dar a conocer todo lo que hace el gobierno, en especial, informar a la población sobre todo aquello que resultaba sospechoso o cuestionable, hasta llegar a la destitución de algunos funcionarios si era necesario. Por otro lado, él sostenía que el parlamento más que una esfera de confluencia de intereses distintos era una arena para la deliberación y la confluencia de opiniones (diversas). En una época en que ya se habían difundido, en sociedades como la inglesa y la francesa, una diversidad de formas de sociabilidad que hacían posible la intercomunicación y la configuración de una "opinión pública" (Sennett 2002, Habermas 2005), Mill encontraba en el parlamento el espacio propicio para la producción discursiva y la discusión sobre asuntos que atañen al conjunto de la nación (Mill [1861] 1991:125) En consecuencia, el parlamento era definido como una esfera en la cual se producían y confluían opiniones diversas; en la que no solo existía una opinión general sino también se reconocían aquellas emanadas desde los contextos locales y donde era posible la controversia y el acuerdo sobre distintas materias.

\section{I.4. Los dilemas sobre el sufragio en cuanto mecanismo de control}

La caracterización que hace Mill acerca del funcionamiento de las instituciones representativas se torna mucho más controvertida al hacer referencia a la participación efectiva del conjunto de la sociedad. En el momento en que Mill reflexiona (1861) el sufragio se había convertido en una importante fuente de poder político y se habían dado algunas experiencias de universalización masculina ${ }^{7}$. La discusión y la preocupación que genera la promoción del sufragio hacia el conjunto de la sociedad, se ligaba con la necesidad que encontraban algunos sectores - incluyendo a los representantes del liberalismo - de garantizar estabilidad y protección del nuevo orden social y político que se estaba constituyendo. De hecho, hacia principios del siglo XIX la necesidad de impulsar mercados desregulados y cautelar el derecho de propiedad llevó a que Jeremy Bentham y James Mill realizasen una fuerte defensa del sufragio de tipo censitario (Bobbio 2001, Macpherson, 1997).

\footnotetext{
${ }^{6} \mathrm{La}$ importancia del elemento discursivo en el liberalismo es reconocido por Matteucci cuando concluye que "una sociedad sin diálogo no es más una comunidad política: ésta, para existir, debe ser en primer lugar una comunidad lingüística de hombres libremente comunicantes. Sólo de esa forma puede autorepresentarse" (Matteucci 2005: 83). ${ }^{7}$ El sufragio universal masculino se instaura en Francia en 1848, con lo cual el número de electores pasa en este país de dos a diez millones (Gaxie 2004: 44).
} 
Sin embargo, la posición de John Stuart Mill respecto al sufragio fue ambivalente y en ocasiones abiertamente contradictoria. Por un lado, planteaba que la alta participación contribuía a mejorar y a educar a las personas. Además, consideraba que los electores encontraban en el sufragio un mecanismo que les permitía escoger entre distintas alternativas y que, a causa de esto mismo, asumía las características de un instrumento de protección frente a las autoridades y al gobierno. Pero por otro lado, sostenía que al estar los electores orientados por un sentimiento egoísta e individualista, una participación masiva de la clase obrera podía llevar a que las decisiones a nivel legislativo se destinen únicamente a beneficiar a este sector.

La posición de Mill frente a la ampliación del sufragio varía también de momento en momento. Mientras en un escrito de 1859 (Ideas sobre la reforma parlamentaria) se manifestaba como un abierto partidario del sufragio universal, en 1861 proponía que debían existir restricciones y diferencias. Se trata de diferencias en la cantidad de votos y en el nivel de influencia entre un sector social y otro, las que se debían dar según el tipo de actividad, el grado de instrucción y la posesión o no de instrucción superior (profesional) (Mill [1861] 1991:204). Al reflexionar sobre la factibilidad del sufragio femenino, decía en su escrito de 1861 que éste podía llegar a ser absolutamente favorable para lograr una mayor autonomía de la mujer, debido a que:"Aprenderá a considerar la política como una cuestión sobre la que se le permite emitir juicio, y en la cual, sino tiene una opinión, debe actuar en favor de ella; adquirirá un sentido de responsabilidad personal en el asunto...” (Ibid:215).

Por tanto, existía en Mill una ambivalencia que, siguiendo a Matteucci (2005), puede ser atribuible al carácter amplio y heterogéneo del liberalismo político. Pero en cierta medida es parte de la tensión que caracteriza a la propia modernidad, que Wagner (1997) identifica en el dilema "libertad" (autonomía) o "disciplina" (control). En Mill esa dicotomía se observaba en la defensa que hacía de la autonomía de los ciudadanos, para lo cual ponía énfasis en los mecanismos de protección y advertía sobre el peligro que encerraba la posibilidad del despotismo y la concentración del poder. Al mismo tiempo, se mostraba partidario de un cierto control y regulación de la misma participación, al establecer diferencias en la capacidad de influencia entre sectores subordinados y aquellos que consideraba con capacidad de ejercer funciones superiores.

Esta tensión se derivaba directamente del vínculo que establecían los representantes con los electores, dada la intención por parte de la clase gobernante de asegurar su plena autonomía respecto a los niveles de influencia de los electores (Pizzorno 1996: 967). De ahí también el retraso o la postergación de la instauración definitiva del sufragio universal, para mantener el control sobre el electorado y aquella población que quedaba excluida de la participación política. 
De La AUTONOMÍA DEL MANDATO A LA RENDICIÓN DE CUENTAS.

Esto demuestra que no bastaba solo con un vínculo sustentado en la confianza, sobre todo si buena parte del poder de decisión y de control era delegado en el representante. No por casualidad, durante el mismo período en que surgen las instituciones democráticas modernas también emergió, desde la propia sociedad, un contrapoder que se expresa en la búsqueda permanente de mecanismos de control sobre los reresentantes ${ }^{8}$.

La existencia de contrapoderes y la institucionalización de mecanismos de control por parte de los ciudadanos, permite como muy bien apunta Rosanvallon (2007:2324), "organizar la desconfianza" en las autoridades, el gobierno y los representantes. A juicio de Rosanvallon, este contrapoder es distinto al de la "democracia electoral". Sin embargo, siguiendo los planteamientos que se han venido presentando desde la teoría de la accountability electoral, el sufragio también puede ser un efectivo mecanismo de control, en la medida en que los electores sean capaces y cuenten con los medios para evaluar la gestión de los representantes y luego sancionar, escogiendo alternativas distintas, o en sus efectos recompensar por medio de la reelección. Por tanto, con la delegación del poder de decisión y la responsabilidad en los representantes, los electores no pierden completamente su soberanía, ya que siguen contando con la posibilidad de ejercitar control sobre el gobierno y el resto de los representantes.

Este hecho es clave para saber qué ocurre una vez que se constituyen las llamadas democracias de masas, como consecuencia de la ampliación del sufragio, la constitución de los sistemas de partidos y la emergencia del profesional de la política durante la segunda mitad del siglo XIX y la primera mitad del XX.

Desde la teoría de la accountability, los ciudadanos pueden asegurar una gestión responsable, junto a respuestas adecuadas de parte de los representantes, a través de un sistema más institucionalizado: la regularidad de las elecciones (Manin 1992, Fisichella 2003). Así, la institucionalización de los mecanismos de control refuerza no solo el vínculo de la representación sino que mantiene el poder de influencia de los electores. Más aun, la institucionalización del control, junto con la organización de la sospecha y de la desconfianza, pueden ser vistos como proyecciones del "derecho de resistencia" surgido durante el medioevo (Rosanvallon 2007: 40) ${ }^{9}$. De manera que es posible señalar que tras el advenimiento de la modernidad, se mantienen algunos as-

\footnotetext{
${ }^{8}$ Según ha señalado recientemente Rosanvallon, desde muy temprano “[...] se ha celebrado la vigilancia del pueblo controlador, perpetuamente activo, como el gran remedio para el funcionamiento de las instituciones, en particular para remediar lo que podríamos llamar la entropía representativa (es decir, la degradación de la relación entre elegidos y electores)" (Rosanvallon 2007: 30).

${ }^{9}$ Rosanvallon se refiere a las teorías acerca de la soberanía popular, surgidas durante el medioevo, que tienen un fundamento divino y religioso. Esta idea de resistencia, reaparece y es reinterpretada durante la reforma protestante - es decir, en una fase pre-liberal -, específicamente en los postulados del calvinismo. Como señala Wolin: "Según Calvino, el ciudadano que, por motivos estrictamente religiosos, desobedecía una orden contraria a las Escrituras, no sólo cumplía su obligación hacia Dios, sino que recordaba al gobernante la verdadera naturaleza de su cargo. La concepción calvinista de la resistencia era la de un servicio desinteresado, que tenía como fin proteger la identidad de las instituciones políticas contra los errores de los funcionarios temporarios” (Wolin 1993: 202).
} 
pectos institucionales de la fase premoderna que influyen en la dinámica asambleísta y parlamentaria, junto a la proyección, bajo otras características, de aquella idea medieval que promueve la resistencia a las tiranías y al mal gobierno.

\section{La representación y el devenir de las democracias contemporáneas}

\section{II.1. La persistencia del dilema liberal}

La centralidad que en un primer momento tienen los procesos electorales se relaciona directamente con la posibilidad de participar en la distribución del poder, debido a que permite a los ciudadanos definir periódicamente la composición del cuerpo legislativo. En efecto, la progresiva universalización del sufragio va permitiendo a los ciudadanos incidir en la naturaleza misma del proceso político. Siguiendo a Poggi (1992: 98-101), tres son las consecuencias más relevantes de esta transformación. En primer lugar, cabe destacar la presencia del partido de masa, sustentado en aspectos ideológicos y doctrinarios, que desplaza al antiguo partido de notables. Con ello se produce el debilitamiento del poder y de la capacidad de control que ciertos grupos sociales - como los terratenientes - ejercían sobre determinados contextos locales y territoriales. En segundo lugar, la competencia partidaria hace posible que ahora se vean reflejados intereses, que en un primer momento quedaban marginados por el peso de determinados grupos sociales. $\mathrm{Si}$ en un primer momento predominan los intereses de aquellos sectores que promueven la mayor liberalización y autorregulación de los mercados, ahora también tienen cabida las opiniones de quienes demandan regulación y proteccionismo estatal. Finalmente, los partidos y grupos organizados pasan a ser determinantes en la definición de aquellas políticas y asuntos de interés nacional. Los representantes y dirigentes derivados directamente de los partidos se ven enfrentados en este nuevo contexto a un importante dilema: actuar de manera autónoma, o actuar en función del mandato asignado por la propia organización, lo que significa en muchos casos tener que decidir de acuerdo a programas o ideologías particulares.

Se produce acá una situación bastante paradójica que afecta al propio representante. Por un lado, en la medida en que su actividad se profesionaliza - es decir, comienza a vivir de la política y no solo para la política - su autonomía respecto de los ciudadanos se ve acrecentada. Pero, por otro, su margen de acción queda restringido a los requerimientos de su organización. Todo ello provoca en el representante una tensión permanente, ya que debe resolver respecto a cómo decidir y a cómo actuar: si en función del interés general o de los aspectos programáticos o de las demandas y aspiraciones de sus electores. Se trata de una proyección de aquella tensión del liberalismo decimonónico que en términos weberianos se expresa entre actuar de acuerdo a la ética de la responsabilidad o, por el contrario, en función de la ética de la convicción. 
De La AUTONOMÍA DEL MANDATO A LA RENDICIÓN DE CUENTAS. UN ALCANCE CONCEPTUAL A LOS MECANISMOS DE REPRESENTACIÓN DEMOCRÁTICA

¿Cómo se resuelve este nuevo dilema? Tomando en cuenta los planteamientos más actuales de la teoría de la representación (Pitkin 1967), una respuesta adecuada consiste en asegurar la articulación entre los ciudadanos y los representantes, a pesar de lo necesario que pasa a ser la autonomía de estos últimos. De hecho, para actuar responsablemente un representante debe en ocasiones hacer caso omiso de las aspiraciones de los ciudadanos, hipotecando con eso su propia "popularidad". Por tanto, es aquí donde pasan a jugar un papel significativo otros agentes que asumen la función de intermediación, y de esa forma hacen posible que el vínculo entre representantes y representados se despersonalice sin que necesariamente se disuelva.

El tratamiento que da Pitkin al problema de la representación destaca por establecer una sugerente distinción entre lo que llama "representación formal" y "representación sustantiva". La "representación formal" se refiere al acto mismo de representar, a partir de la presencia de mecanismos que hacen posible la transmisión del mandato y el proceso de control o rendición de cuentas (accountability). La de "contenido", en cambio, comprende aspectos que se ubican en el plano simbólico, de reproducción de "imágenes" y la actuación en función de las sensibilidades de los representados. Dicho en otros términos, la primera alude a la responsabilidad del representante, la segunda en cambio a su capacidad de respuesta.

Para la primera forma de representación, Pitkin reinterpreta a Hobbes y a Burke, señalando que las decisiones de los representantes deben estar orientadas en pos de los intereses de la nación, sin por eso descuidar la defensa de los intereses de las localidades o provincias por la cuales fueron electos ${ }^{10}$. Siguiendo a Burke, sostiene que le corresponde al representante comunicar respecto a lo que acontece en el sistema político, agregando a ello un componente adicional, al sostener que el representante puede justificar sus decisiones, cuando éstas son contrarias a determinados intereses.

La segunda forma de representación depende muchas veces del carácter vinculante, sentido de pertenencia y nivel de identificación, que expresen los propios ciudadanos. Por el contrario, no existe representación de "contenido" o "sustantiva" si las decisiones se dejan a expertos o se adoptan de manera arbitraria (Ibid:212). De ahí por tanto la importancia que alcanzan las instituciones y órganos de intermediación: mass media, sindicatos, partidos u otro tipo de organizaciones sociales. Son estas instituciones las que facilitan el proceso de comunicación y justificación, respecto a una determinada decisión, ayudando además en el plano simbólico a fortalecer el sentido de pertenencia y de identificación.

Para el funcionamiento de la representación democrática, Pitkin considera gravitante la idea de "responsabilidad". La gestión de un gobierno puede perfectamente estar

\footnotetext{
${ }^{10}$ En palabras de Pitkin: "El representante es, típicamente, defensor y juez, un agente de su localidad y un gobernante de la nación. Su deber es perseguir los intereses locales y nacionales, lo primero porque es un representante, lo otro porque su labor como representante es gobernar la nación. Esta doble función es dificultosa, pero no es ni prácticamente ni teóricamente imposible" (Ibid: 218).
} 
orientada hacia mayores niveles de bienestar para la población. Sin embargo, esto no necesariamente implica que se esté en presencia de acciones "responsables" ni tampoco de una democracia representativa, pues un gobierno autoritario puede perfectamente garantizar u otorgar dicho objetivo (Ibid:231). De ahí la crucial importancia que tiene la autonomía y la independencia de las decisiones de un representante, siempre y cuando las decisiones sean justificadas y, a la vez, reconocidas por parte de los representados.

\section{II.2. La importancia de la competencia}

La propuesta de Pitkin puede ser interpretada como un intento de dar respuesta a una serie de discusiones que se vienen dando al interior de la teoría democrática contemporánea. Si se toma en cuenta lo que ha sido la discusión a partir de la segunda guerra mundial, es posible observar la aparición - o mejor dicho reaparición - de la crítica a la democracia representativa como expresión del elitismo político por parte de quienes apuestan a promover formas mucho más participativas. Este aspecto se ve complementado por la contraposición que, muchas veces, se tiende a realizar de las dinámicas que se dan al interior del sistema político, respecto de aquellas presentadas en la sociedad civil.

Lejos de exacerbar el elitismo, más bien lo que se produce en el caso de la competencia política es una forma mucho más clara de asegurar respuesta y responsabilidad de parte del conjunto de los representantes. Incluso más, determinadas instituciones de la sociedad civil como los grupos de interés y los mass media, al establecer canales de comunicación e interacción con los actores en competencia, asumen un importante rol de complemento, contribuyendo a hacer efectivo dicho proceso. Así como el representante debe informar para justificar muchas de las decisiones, también debe establecer un canal de comunicación continuo para hacer de la competencia un proceso permanente que vaya más allá de los eventos electorales, demostrado de esa forma su capacidad de control y fiscalización (Dahl [1966] 1976, Pasquino 2005).

Tanto en Schumpeter como en Dahl, existe un fuerte énfasis por delimitar la arena política en función de los alcances que tiene el proceso competitivo. Eso hace que muchas veces la propia noción de democracia, propuesta por ambos autores, sea interpretada en modo restringido o asociada simplemente a los elementos procedimentales que definen una parte del proceso democrático. Sin embargo, ellos mismos reconocen que la democracia es una precondición de la competencia, ya que para que existan "mercados electorales" abiertos y plurales deben estar garantizadas una serie de condiciones mínimas, tales como la libertad de información, de reunión y asociación, así como también la posibilidad para asegurar una alta convocatoria de las elecciones. Siguiendo a Dahl (1971), estas condiciones posibilitan el desarrollo de 
De LA AUTONOMÍA DEL MANDATO A LA RENDICIÓN DE CUENTAS. UN ALCANCE CONCEPTUAL A LOS MECANISMOS DE REPRESENTACIÓN DEMOCRÁTICA

una competencia abierta y, por ende, la generación de una mayor pluralidad, lo cual permite a su vez una ampliación de las estructuras de representación.

En la definición de Dahl, el proceso competitivo va más allá de la mera disputa por el poder, o por alcanzar determinadas cuotas del poder político, pues la competencia y en particular aquella "contestación” que estimula la presencia de fuerzas políticas opositoras, permiten asegurar responsabilidad y respuesta por parte de los representantes que se encuentran en el gobierno. En este sentido, la ausencia de una adecuada "contestación" o de una débil oposición, producen un efecto contrario, llevando incluso a que se pierda el sentido de la participación electoral. Esta constatación es compartida por aquellos autores que ven en la responsabilidad política un componente inherente de toda representación de carácter electa (Manin 1992, Sartori 1995 , Cedroni 2004), y también por quienes logran diferenciar la representación adscrita netamente al sistema político de otras que emergen en la sociedad civil (Bobbio 1988, Fisichella 1996).

Una baja competencia política, o la presencia de un sistema de partidos débilmente institucionalizado, no solamente merman la posibilidad de control de la representación electiva sino también la canalización de las demandas sociales ${ }^{11}$. De hecho, el debilitamiento de los partidos de masa en la segunda mitad del siglo XX significó en muchos casos una "invasiva penetración de intereses privados" hacia las decisiones públicas, producto de una mayor presión de los grupos organizados desde la sociedad civil (Cedroni 2004: 12). Al respecto Pitkin ha señalado - con un sentido altamente realista - que el representante no puede hacer abstracción de las realidades locales, ni mucho menos de las aspiraciones de sus representados. En esta misma línea, Bobbio dice que la "representación política" puede ser una "representación de intereses" en la medida que existan mecanismos y condiciones institucionales para resolver acerca de los “intereses generales" (Bobbio 1988: 11).

En consecuencia, la competencia juega un doble rol en el sentido que permite, por un lado, canalizar las demandas sociales y, junto a ello, hacer efectiva la institucionalización democrática (Dahl 1971, Bartolini 1999, Gaxie 2004). Dicha institucionalización se evidencia con claridad a partir del uso efectivo y en el reconocimiento que hacen los distintos actores sociales y políticos, de las principales arenas de participación y deliberación democrática. Las limitaciones que presente el sistema político, en materia institucional, harán que los intereses sean canalizados por una esfera alternativa, como puede ser la sociedad civil. Además, tales limitaciones tendrán como consecuencia la irrupción y el excesivo predominio de los intereses particulares, dificultando con ello las posibilidades de consenso y acuerdo.

\footnotetext{
${ }^{11}$ Un sistema representativo abierto y competitivo permite la regulación e institucionalización del conflicto, debido a que se encuentra en condiciones de realizar una mayor absorción de las demandas que emanan desde la sociedad (Bartolini 1999: 443).
} 


\section{II.3. El significado de la rendición de cuentas y la importancia del proceso electoral}

El fortalecimiento de las instituciones democráticas conlleva la necesidad de promover y consolidar mecanismos para la rendición de cuenta (accountability). Tales mecanismos involucran a un conjunto de actores e instituciones, pasando por algunas instituciones estatales - como los tribunales de justicia y las asambleas legislativas -, los actores de la competencia política - electores y partidos - y las organizaciones de la sociedad civil-mass media, grupos de interés, organizaciones de derechos humanos, organizaciones no gubernamentales, entre otras. La rendición de cuenta es definida como la responsabilidad (responsibility) que tienen los representantes frente a sus representados, las instituciones públicas y otras derivadas de la sociedad civil (Schedler 1999, Diamond y Morlino 2005, Bovens 2006, O’Donnell 2007) ${ }^{12}$. En ocasiones, la acción de los representados y las instituciones posibilita también que los representantes y las autoridades que han sido electas puedan responder a las expectativas de los representados (responsiveness).

En los procesos de rendición de cuenta, tanto actores políticos como instituciones pueden ejercitar control y en ocasiones sancionar la gestión de los representantes. Para ello es necesario que se den algunas condiciones, como una adecuada separación de poderes y la presencia de un sistema político institucionalizado. Del mismo modo, también es necesario el fortalecimiento de las organizaciones de la sociedad civil y la disposición de los ciudadanos de participar en ellas. Los actores y las instituciones difieren entre sí, en términos de funciones y en el tipo de control que pueden llegar a ejercitar. Así por ejemplo, los electores eventualmente evalúan la gestión de los representantes para luego decidir a través del voto si sancionar o recompensar a los representantes (Diamond y Morlino 2005, O'Donnell 2007). Asimismo, las instituciones públicas, mass media u otras organizaciones de la "sociedad civil", hacen efectivo el control por medio del monitoreo sobre representantes que han sido electos o no electos (como por ejemplo ministros u otros funcionarios nominados desde el ejecutivo). Por tanto, al interior de un sistema democrático pueden interactuar diferentes formas de accountability que se diferencian en términos de sus actores, procedimientos y resultados. En buenas cuentas, al interior de un sistema democrático, los procesos de rendición de cuenta (accountability) atenúan los problemas derivados de la creciente autonomía asumida por el sistema político, contribuyendo de este modo a reforzar su carácter representativo.

\footnotetext{
${ }^{12}$ Según Bovens (2006: 6-7), el uso del concepto de accountability se remonta al año 1066, inmediatamente después de la invasión normanda en Inglaterra, fecha en la cual el rey Guillermo I comienza a usar el término "a count" con el fin de obtener información sobre los bienes de los propietarios. Posteriormente, en la tradición anglosajona se utiliza el concepto para entregar información sobre el estado económico y financiero del gobierno. Siempre en la tradición anglosajona, desde los años ochenta - del siglo pasado - el concepto de accountability emerge con la finalidad de evaluar la gestión gubernamental. Como agrega Bovens, en la actualidad accountability connota al mismo tempo instrumento y meta. Se inicia como instrumento, con el fin de hacer eficiente y eficaz la gestión del gobierno, pero finaliza como una meta en sí misma.
} 
De La AUTONOMÍA DEL MANDATO A LA RENDICIÓN DE CUENTAS. UN ALCANCE CONCEPTUAL A LOS MECANISMOS DE REPRESENTACIÓN DEMOCRÁTICA

Cabe señalar que existen definiciones que apuntan a destacar si el tipo de relación que se produce entre actores e instituciones es asimétrica u horizontal. Diamond y Morlino (2005: xix-xxv) y con más detalle O’Donnell (2007, cap. II, III y IV), hablan de accountability vertical, cuando las relaciones son asimétricas, como ocurre normalmente entre electores y representantes electos $\mathrm{u}$ organizaciones sociales y miembros del ejecutivo. En cambio la accountability horizontal, derivada de la tradición "madisoniana" del check and balance y de la división de poderes, se refiere a la relación entre instituciones como parlamento y gobierno; contraloría y administración pública; tribunales de justicia y parlamento o tribunales de justicia y ejecutivo. Otras distinciones, derivadas de estas mismas definiciones, plantean la diferencia entre los procesos de: i) accountability electoral, cuando se hace solo referencia a situaciones que ocurren a nivel de las dinámicas electorales; ii) social, para destacar la capacidad de monitoreo y de control desde la sociedad civil; iii) e interinstitucional cuando, como en el caso de la horizontal, se alude a la fiscalización, información y sanción que se produce entre las instituciones y poderes del Estado.

Independiente de la forma de relación y de los actores que participan, así como los representados llevan a cabo el monitoreo y el control, corresponde a los representantes informar y justificar respecto de su desempeño (Schedler 1999: 14). Tal como lo señala Pitkin, lo relevante en un sistema representativo no es responder directamente a las demandas e intereses de los representados, sino más bien actuar de manera responsable justificando e informando a estos últimos.

Entre las distintas formas de accountability, la relación suele ser de complemento y no de rivalidad. Sin embargo, es posible plantear que existe mayor centralidad de los procesos electorales respecto de otras dinámicas que se dan a nivel de las instituciones públicas o al interior de la sociedad civil. En primer lugar, los procesos electorales pueden determinar el tipo de funcionamiento de una parte de los procesos de accountability horizontal o interinstitucional, en especial en lo que se refiere al rol del parlamento y a las funciones de fiscalización y control de parte de la oposición. Si la competencia electoral es limitada, la contestación y desempeño de la oposición al interior del parlamento será débil y hasta insignificante. En segundo lugar, es mucho más factible pensar la institucionalización a nivel del sistema político que en la sociedad civil, teniendo en cuenta la enorme diferenciación y heterogeneidad que existe en esta última esfera.

En términos temporales, la accountability que se genera desde lo electoral es interpretada como un proceso, o mejor dicho una cadena de procesos, que hacen posible que los electores puedan evaluar a un representante en dos sentidos: uno retrospectivo y otro prospectivo (Campus 2000, Pasquino 2005). La evaluación retrospectiva se manifiesta a través de la evaluación que los electores hacen del desempeño y la calidad de la gestión de los representantes. Apela directamente al pasado y a la capacidad que los 
electores tengan para reconocer las fortalezas y las debilidades de lo que ha sido una experiencia representativa. La evaluación prospectiva, en cambio alude al futuro, ya que toma como referencia los aspectos programáticos y las ofertas de quienes serán los representantes, incluyendo por cierto a quienes aspiran ser reelectos.

En función de estos dos tipos de evaluaciones, o de una por sobre la otra, los electores finalmente deciden sancionar o recompensar. Sin embargo, todo esto presupone, en primer lugar, que las decisiones que adoptan los electores son netamente racionales (Manin, Przeworski y Stokes 1999). En segundo lugar, presupone también que las decisiones se efectúan sobre la base de criterios utilitaristas e individualistas. A raíz de lo mismo, son necesarias algunas condiciones para garantizar el funcionamiento de este modelo, como la presencia de electores altamente informados y una comunicación permanente emanada desde el sistema político. En ese sentido, el rol de los partidos y de los medios de comunicación pasan a ser fundamentales, en cuanto asumen el papel de actores protagónicos del proceso competitivo, ya que la "lucha por las preferencias" de los electores incentiva una mayor comunicación política.

Un primer reparo a esta interpretación (Campus 2000: 44) sostiene que los electores se tienden a interesar e informar sobre lo que ocurre en el sistema político principalmente en los momentos previos a las elecciones, pues en esos momentos aumenta el flujo de información que emana desde el gobierno y los partidos. Pero existen otras críticas que se dirigen a los supuestos racionales de las decisiones de los electores. Al respecto, Pizzorno considera dificultoso que los electores puedan escoger en función de programas diversos (Pizzorno 1994: 148). Según explica, los electores generalmente deciden sin estar informados y a su vez son limitadas las posibilidades de que éstos puedan evaluar en base a una multiplicidad de variables y de factores probablemente externos ${ }^{13}$. Por otra parte, especial importancia le asigna a la dimensión simbólica del proceso político, en particular al fenómeno de la ceremonialidad, ya que las elecciones para muchas personas son simplemente ritos (Ibid: 160). A ello se agregan otro tipo de factores no racionales que responden al peso de la tradición o al sentido de pertenencia a determinados grupos sociales. En relación a este punto, Pitkin señala que la adopción de determinadas preferencias puede venir del contacto y la influencia de los grupos primarios, como familia y amigos (Pitkin 1967: 119). Destaca además la importancia que pueden tener los factores emocionales, en especial, la sintonía de ciertos sentimientos con las apelaciones o discursos de candidatos, partidos y líderes políticos en general (Ibid: 92ss).

Sobre la base de estas críticas, resulta oportuno sostener que la decisión electoral combina elementos utilitarios o racionales con factores de orden simbólico y de tipo vinculante, sean afectivos o identitarios. Por cierto, el predominio de uno u otro factor, en la decisión final dependerá de la forma en que los electores procesen

\footnotetext{
${ }^{13}$ "Si el elector es un agente racional - pregunta Pizzorno -, ¿ jor qué asume un acto tan irracional como ir a votar? Esto, como se puede fácilmente calcular, no produce ninguna utilidad...” (Pizzorno 1994: 150).
} 
De La AUTONOMÍA DEL MANDATO A LA RENDICIÓN DE CUENTAS. UN ALCANCE CONCEPTUAL A LOS MECANISMOS DE REPRESENTACIÓN DEMOCRÁTICA

la comunicación política o el flujo comunicativo que se produce antes y durante las elecciones (Campus 2000: 20-21). Más específicamente, dependerá del nivel de preparación e instrucción de los electores, sus motivaciones e intereses y, finalmente, de las oportunidades que pueda ofrecer el propio sistema político. De este modo, los electores podrán buscar información para escoger a un determinado candidato o partido, o bien, reafirmar sus opiniones preexistentes.

\section{II.4. El debilitamiento y la crisis de la representación en las últimas décadas}

Como se ha venido insistiendo, la representación democrática se manifiesta a través de un vínculo despersonalizado que hace posible la autonomía del mandato en la figura del representante. El traspaso de una parte del poder y de las decisiones hacia este último lleva históricamente a la búsqueda de una serie de mecanismos que permitan, por un lado, ejercer un determinado control y, en consecuencia, asegurar responsabilidad. De esa forma, se logra mantener la representatividad como principio fundante de la propia democracia. Como se ha visto, los procesos electorales cumplen una función significativa, en la medida que permiten elegir a los representantes, aseguran una distribución del poder - si las elecciones son competitivas y abiertas - y promueven la evaluación de su gestión. Pero en las últimas décadas se han desencadenado una serie de fenómenos, originados muchas veces al interior del sistema político, que tienden a erosionar los fundamentos de la representación, atentando incluso contra la sostenibilidad de la propia democracia.

Varios de estos fenómenos han sido evidenciados en la experiencia de las "nuevas democracias", atribuyéndolos a las dificultades que se fueron generando en los procesos de transición y consolidación. Sin embargo, también aparecen en aquellas democracias surgidas con posterioridad a la segunda posguerra. En el caso particular de las "nuevas democracias" latinoamericanas, los fenómenos más significativos dicen relación con las escasas garantías para la división y autonomía de los poderes del Estado - atribuidos normalmente al marcado presidencialismo -, trayendo consigo un aumento de las atribuciones del ejecutivo y escasas o poco significativas funciones del parlamento. Por ende, desde principios de los años noventa se utiliza el concepto de "democracia delegativa" para caracterizar regímenes democráticos que carecen de una adecuada representación, debido a la ausencia de los mecanismos de control y al carácter pasivo de la ciudadanía (O’Donnell 1994). Este concepto da cuenta de la existencia de democracias en las que, junto a la concentración de poderes, se gobierna a partir del uso permanente de decretos para eludir el control del parlamento, y donde existen sistemas de partidos fragmentados y con baja legitimidad. También se suelen implementar políticas radicalmente distintas a las contenidas en el programa original. 
Tal situación, junto a los síntomas que han presentado algunas democracias europeas en los años noventa, lleva a un conjunto de autores a difundir el uso de los conceptos antipolítica y neopopulismo (Mayorga 1995, Campus 2006, Mainwaring 2006). La antipolítica se relaciona con la emergencia de un discurso cuyo contenido pone énfasis en los componentes tecnocráticos de la gestión gubernamental, el cual se acompaña de una fuerte crítica al rol de los partidos y demás formas de intermediación, como ocurre con los sindicatos. El neopopulismo, por su parte, se define primero como discurso y luego como práctica. Como discurso, manifiesta una notoria diferencia con el "populismo clásico" por el hecho de que su contenido es marcadamente antiestatista y dogmáticamente neoliberal. Pero como práctica, el neopopulismo posee una serie de características que se confunden con los contenidos del discurso de la antipolítica, entre ellas: i) la emergencia de liderazgos outsider, es decir, de figuras sin una trayectoria política previa - normalmente vinculados al mundo empresarial - que irrumpen en épocas de crisis, con discursos mesiánicos o simplemente promotores de reformas radicales - como Collor de Mello en Brasil, Fujimori en Perú, o Berlusconi en Italia -; ii) el reemplazo de la función de las organizaciones intermediarias por un contacto directo con las masas, y por un fuerte personalismo que se asume a través de un excesivo protagonismo de los mass media iii) y el uso de recursos que eluden la discusión y la deliberación de las instituciones representativas, como el de los plebiscitos y los decretos "con fuerza de ley".

Cualquiera sea el concepto, lo cierto es que en experiencias que puedan ser definidas como neopopulistas, o donde se impone el discurso de la antipolítica, subyacen la ausencia de condiciones de pluralidad, debido a una situación de concentración mediática o control sobre los medios de comunicación, escasa contestación debido a la fragmentación del sistema de partidos o restricciones para su funcionamiento y, nulas posibilidades de ejercitar control sobre la gestión de los representantes. En el caso particular de las elecciones, éstas dejan de ser un mecanismo al servicio de los electores transformándose más bien en un instrumento de manipulación de los representantes.

\section{Conclusiones}

La separación y autonomización de la representación política constituyen sin duda una tendencia irreversible en la trayectoria de los sistemas democráticos. Es por ello que resulta relevante la presencia de mecanismos e instrumentos que aseguren un cierto nivel de control, lo cual deviene en una necesidad, e incluso en un principio inherente, para el funcionamiento mismo de la democracia. De este modo, la idea de representación autónoma que es promovida desde finales del siglo XVIII se distancia de aquella prevaleciente en la fase premoderna. Pero al mismo tiempo, se distancia 
de aquellas concepciones de la política que, no obstante su carácter moderno, abogan más bien por una regulación y moderación del poder por parte de quienes lo detentan, como se conoce bajo la fórmula de Maquiavelo.

Los sistemas representativos dependen de la capacidad que desarrollen para articular autonomía y control, pero esta articulación no resulta fácil. Más bien en ocasiones deviene en una verdadera tensión, si se piensa que la autonomía del representante puede ser interpretada como la separación o divorcio definitivo con el resto de la sociedad. Una consecuencia clara de esta separación son las manifestaciones de desafección hacia el sistema político que se producen al interior de varias de las democracias contemporáneas, incluyendo las más avanzadas, frente a lo cual cabe una enorme importancia al sentido vinculante que puede derivar de los pocesos de competencia entre partidos, siempre y cuando ésta sea abierta, plural y con una oferta programática lo suficientemente amplia. Al interior de los sistemas democráticos, la presencia de condiciones y garantías de pluralidad facilita la institucionalización de determinadas formas de control que aseguren responsabilidad y respuesta por parte de los representantes. Son diversos los autores que tienden a reconocer un aumento en las condiciones de pluralidad, a partir de transformaciones estructurales y globales más recientes, como la expansión del uso de las tecnologías de la información y la comunicación. Dicha pluralidad se expresa a través de la proliferación de formas de participación al interior de la sociedad civil, así como también, por efecto de la expansión de las tecnologías de la comunicación, en la mayor presencia de comunidades virtuales. Sin embargo, como se ha señalado en este artículo, resulta más factible y viable pensar en las posibilidades de control que ofrece la propia competencia entre partidos y de una amplia participación en los procesos electorales, considerando también que ésta sea combinada con formas de participación más directa, como plebiscitos y referéndum. En la medida en que las elecciones alcancen un carácter vinculante, se estará en presencia de una clara aceptación de las reglas del juego democrático de parte de los ciudadanos; $y$, a su vez, e independiente del sentido asignado al uso del sufragio - utilitario o emocional - frente a posibilidades de transformación del entorno institucional, en virtud de los resultados de la competencia. 


\section{Bibliografia}

Accarino, B. (1999): Rappresentanza. Il Mulino, Bologna.

Bartolini, S. (1999): "Collusion, competition and democracy". En: Journal of Theoretical Politics, 11 (4), pp. 435-470.

Bobbio, N. (1998): "Rappresentanza e interessi”. En: Gianfranco Pasquino (comp.): Rappresentanza e democrazia, Laterza, Roma-Bari, pp. 1-27.

Bobbio, N. (2001): Liberalismo y democracia. Fondo de Cultura económica, México.

Bovens, M. (2006): “Analysing and assesing public accountability. A conceptual framework”. En: European governance papers, 1, enero, pp. 1-37.

Burke, E. (1774, trad., 1984): “Discurso a los electores de Bristol”. En Textos políticos, Fondo de Cultura Económica, México, pp.311-314.

Campus, D. (2000): L'elettore pigro. Informazione politica e scelta di voto. Il Mulino, Bologna.

Campus, D. (2006): L’antipolitica al governo. Il Mulino, Bologna.

Cedroni, L. (2004): La rappresentanza politica. Teoria e modelli. Franco Angelli, Milano.

Cotta, M. (1986): “Parlamenti e rappresentanza”. En Gianfranco Pasquino (comp.): Manuale di Scienza Politica, Il Mulino, Bologna, pp. 281-328.

Dahl, R. (1966, trad.,1976) :"Pattern of opposition”. En Robert Dahl (comp.): Political oppositions in Westerm democracies, Yale University, pp. 332-347.

Dahl, R. (1971): Polyarchy. Participation and opposition.Yale University Press, New Haven.

Diamond, L. y Morlino, L. (2005): "Introduction". En: Assesing the quality of democracy, The John Hopkins University Press, Baltimore, 2005, pp. ix-xliii.

Fisichella, D, (1996): La rappresentanza politica. Laterza, Roma-Bari.

Fisichella, D. (2003): Elezioni e democrazia. Un'analisi comparata. Il Mulino, Bologna.

Gaxie, D. (2004): La democracia representativa. Ediciones Lom, Santiago, 2004.

Groethuysen, B. (1993): Filosofía de la Revolución francesa. Fondo de Cultura Económica, México 1993.

Habermas, J. (2005): Storia e critica della opinione pubblica. Laterza, Roma-Bari. 
De LA AUTONOMÍA DEL MANDATO A LA RENDICIÓN DE CUENTAS. UN ALCANCE CONCEPTUAL A LOS MECANISMOS DE REPRESENTACIÓN DEMOCRÁTICA

Macpherson, C. B. (1997): La democracia liberal y su época. Alianza Editorial, Madrid, 1997.

Mainwaring, S. (2006): "The crisis of representation in the Andes". En: Journal of democracy, Vol. 17, N³, pp. 13-27.

Manin, B. (1992): La democrazia dei moderni. Edizioni Anabasi, Milano.

Manin, B., Przeworski, A. y Stokes, S. (1999): “Elections and representation”. En Democracy, accountability, and representation, Cambridge University Press, pp. 29-54.

Mann, M. (1991): Las fuentes del poder social, I, Una historia del poder desde los comienzos hasta 1760 d.C.Alianza Universidad, Madrid.

Matteucci, N. (2005): Il liberalismo. Il Mulino, Bologna.

Mayorga, R. (1995): Antipolítica y neopopulismo. Cebem, La Paz-Bolivia.

Mill, J. S. (1861, trad. 1991): Consideraciones sobre el gobierno representativo, Gernika, México.

O’Donnell, G. (1994): “Delegative democracy”. En: Journal of democracy, Vol. 5, N¹, pp. 155-69.

O’Donnell, G. (2007): Disonancias. Críticas democráticas a la democracia. Prometeo Libros, Buenos Aires, 2007.

Pasquino, G. (2005): “The quality of democracy”. En: Working Paper, No 2-3, Centre for the Study of Democratic Government, University of Oxford, pp. 1-24.

Pitkin, H. F.(1967): The concept of representation. University of California Press, Berkeley, 1967.

Pizzorno, A. (1994): Le radici della politica assoluta e altri saggi. Feltrinelli, Milano.

Pizzorno, A. (1996): "Mutamenti nelle istituzioni rappresentative e sviluppo dei partiti politici”. En: Paul Bairoch y Eric J. Hobsbawm (comp.): Storia d'Europa, Vol. 5, L'età contemporanea, Einaudi, Torino, pp. 961-1031.

Poggi, G. (1978): La vicenda dello stato moderno. Il Mulino, Bologna.

Poggi, G (1992): Lo stato. Natura, sviluppo, prospettive. Il Mulino, Bologna.

Rosanvallon, P. (2007): La contrademocracia. La política en la era de la desconfianza. Ediciones Manantial, Buenos Aires.

Sartori, G. (1995): “Rappresentanza” en Elementi di teoria politica. Il Mulino, Bologna, pp. 285-327. 
Schedler, A. (1999): “Conceptualizing accountability". En: Andreas Schedler, Larry Diamond y Marc F. Plattner (comp.): Power and accountability in new democracies, Bouldner, Londres, pp. 13-28.

Sennett, R. (2002): El declive del hombre público. Ediciones Península, Barcelona.

Wagner, P. (1997): Sociología de la modernidad. Libertad y disciplina. Herder, Barcelona.

Wolin, S. (1993): Política y perspectivas. Continuidad y cambio en el pensamiento político occidental.Amorrortu, Buenos Aires. 